\section{Clopidogrel resistance in dual antiplatelet therapy after carotid stenting}

We read with great interest the article by Chamraoui et $\mathrm{al}^{1}$ reporting about their favorable experience with the use of ticagrelor as part of dual antiplatelet therapy (DAPT) in 67 patients undergoing transcarotid artery revascularization (TCAR).

DAPT is the key treatment for patients undergoing carotid stenting, and current therapeutic regimens include a combination of acetyl-salicylic acid and a thienopyridine, the most used of which is clopidogrel. ${ }^{2}$ However, the pharmacologic "resistance" to the effect of thienopyridines, which can affect about 30\% of patients taking that drugs, ${ }^{3}$ may increase the risk of periprocedural neurologic events and stent thrombosis.

The effect of alternative therapies have been studied mostly on patients with acute coronary syndrome undergoing coronary stenting, for whom ticagrelor has shown greater antiplatelet efficacy compared with clopidogrel. ${ }^{4}$

There are currently few studies on patients undergoing carotid stenting, for which the presence of an optimal DAPT aggregation is equally fundamental.

In their retrospective experience, Ghamraoui et $\mathrm{al}^{\top}$ did not observe major bleeding events or cerebrovascular ischemic events or death at 30 days postoperatively with the use of ticagrelor after TCAR. Therefore, the authors concluded that ticagrelor may represent a safe and effective alternative to overcome clopidogrel nonresponsiveness in DAPT regimens for TCAR.

Nevertheless, a control group of patients undergoing conventional DAPT regimen with clopidogrel is lacking. Ticagrelor has a well-known low rate of on-treatment platelet reactivity, ${ }^{5}$ but its routine use in patients in whom clopidogrel may be properly effective may raise some cost issues. We agree with the authors about the need for a tailored approach in patients who should undergo DAPT after carotid stenting, but in our opinion this goal could be accomplished by performing platelet reactivity testing as part of routine care, and switching to ticagrelor in the event of thienopyridine resistance.

In our opinion, further studies are needed in the overall population of patients undergoing carotid stenting, to bring evidence either in favor or against the use of ticagrelor as an alternative therapy in clopidogrel nonresponsiveness.

\section{Daniela Mazzaccaro, MD, PhD}

Operative Unit of Vascular Surgery

IRCCS Policlinico San Donato

San Donato Milanese

Milan, Italy

\section{Marco Ranucci, MD, PhD}

Department of Cardiothoracic, Vascular Anesthesia and Intensive Care

IRCCS Policlinico San Donato

San Donato Milanese

Milan, Italy

\section{Giovanni Nano, MD}

Operative Unit of Vascular Surgery

IRCCS Policlinico San Donato

San Donato Milanese and

Department of Biomedical Sciences for Health

University of Milan

Milan, Italy

\section{REFERENCES}

1. Ghamraoui AK, Ricotta JJ 2nd. Outcomes and strategy of tailored antiplatelet therapy with ticagrelor in patients undergoing transcarotid artery revascularization. J Vasc Surg 2021;73: $132-41$.

2. Ricotta JJ, Aburahma A, Ascher E, Eskandari M, Faries P, Lal BK Society for Vascular Surgery. Updated Society for Vascular Surgery guidelines for management of extracranial carotid disease. J Vasc Surg 2011;54:e1-31.

3. Di Dedda U, Ranucci M, Baryshnikova E, Castelvecchio S; Surgica and Clinical Outcome Research Group. Thienopyridines resistance and recovery of platelet function after discontinuation of thienopyridines in cardiac surgery patients. Eur J Cardiothorac Surg 2014:45:165-70.

4. Barkat M, Hajibandeh S, Hajibandeh S, Torella F, Antoniou GA. Systematic review and meta-analysis of dual versus single antiplatelet therapy in carotid interventions. Eur J Vasc Endovasc Surg 2017;53: 53-67.

5. Lemesle G, Schurtz G, Bauters C, Hamon M. High on-treatment platelet reactivity with ticagrelor versus prasugrel: a systematic review and meta-analysis. J Thromb Haemost 2015;13:931-42.

\section{https://doi.org/10.1016/j.jvs.2021.04.076}

\section{Increased rates of ruptured abdominal aortic aneurysm during the COVID-19 pandemic}

A significant reduction in arterial case volume entry has been reported by Natarajan et al in their recent article. Screening for abdominal aortic aneurysm (AAA) in the general population and follow-up of patients with small AAA have been postponed. ${ }^{2}$ The downstream consequences of delaying and reducing vascular visits and procedures remain undetermined. We have analyzed the reasons for the increased number of operations for ruptured AAA during the pandemic period (March 2020-April 2021) in our hospital, which is the only tertiary referral center in the province of Pavia (Lombardy) with 545,000 inhabitants. In the last 5 years, the population has been stable for number and mean age. Data of patients who had surgery for ruptured AAA during the pandemic period were prospectively collected and compared with those of patients who had surgery in the same period of the previous year. Elective and emergency arterial operations were reduced during the pandemic period (overall arterial operations, excluding accesses for dialysis, from 1000 to 630; emergency arterial operations from 200 to 160). The number of operations for ruptured AAA increased either for number or for percentage of emergency operations (12/200; $6 \%$ to 18/160; 11.2\%). In the pandemic period, screening for AAA in the general population was not performed. Almost no patient without symptoms or with mild symptoms was referred for abdominal ultrasound or 